Quim. Nova, Vol. 36, No. 9, 1288-1295, 2013

\title{
FRAÇÕES DA MATÉRIA ORGÂNICA E PROPRIEDADES REDOX DE SUBSTÂNCIAS HÚMICAS EM SEDIMENTOS DE OCEANOS PROFUNDOS
}

\author{
Marihus Altoé Baldotto* \\ Setor de Química e Fertilidade do Solo - Campus de Florestal, Universidade Federal de Viçosa, Rodovia LMG 818, km 6, \\ 35690-000 Florestal - MG, Brasil \\ Arizoli Antonio Rosa Gobo, Marcos Sarmet Moreira de Barros Salomão e Carlos Eduardo Rezende \\ Centro de Biociências e Biotecnologia, Universidade Estadual do Norte Fluminense Darcy Ribeiro, Av. Alberto Lamego, 2000, \\ 28013-602 Campos dos Goytacazes - RJ, Brasil \\ Plínio Barbosa de Camargo \\ Centro de Energia Nuclear na Agricultura, Universidade de São Paulo, Av. Centenário 303, 13416-000 Piracicaba - SP, Brasil
}

Recebido em 8/1/13; aceito em 27/5/13; publicado na web em 5/7/13

\begin{abstract}
FRACTIONS OF ORGANIC MATTER AND REDOX PROPERTIES OF HUMIC SUBSTANCES IN SEDIMENTS FROM DEEP OCEANS. Previous studies have verified that free radicals such as quinone moieties in organic matter participate in the redox reactions in natural systems. These functional groups were positively correlated with the increase in aromaticity and hydrophobicity of the humic substances. As an alternative to relatively complex and expensive spectroscopic methods, the redox properties of the humic substances, determined by potentiometric titrations, have been used to evaluate organic carbon stability in soil and sediments. The present study aimed to perform organic matter fractionation and isolation of humic substances from deep oceans in different isobaths $(750 ; 1,050 ; 1,350 ; 1,650 ; 1,950 \mathrm{~m})$ to determine their redox properties by iodimetric titrations under an inert atmosphere and specified conditions of $\mathrm{pH}$ and ionic strength. Sediment samples were collected to the North and South of platforms of petroleum exploration located in the North of Rio de Janeiro State, Brazil. Fractions of organic carbon and redox properties of humic substances varied with origin and depth of the samples and with position North and South of the petroleum exploration area.
\end{abstract}

Keywords: sediments; organic carbon; electrochemistry.

\section{INTRODUÇÃO}

O maior compartimento da matéria orgânica (MO) de solos, águas e sedimentos ocorre na forma de substâncias húmicas $(\mathrm{SH})$, uma mistura heterogênea de compostos orgânicos agregados por interações fracas de natureza hidrofóbica e por pontes de hidrogênio. As SH podem ser classificadas, operacionalmente, em função da solubilidade em meio aquoso, em ácidos fúlvicos, ácidos húmicos ou huminas. Os ácidos fúlvicos apresentam quantidade de grupos hidrofílicos suficientes para permanecerem solúveis em qualquer valor de $\mathrm{pH}$ e os ácidos húmicos, mais hidrofóbicos, apresentam agregação e precipitação em meio ácido. ${ }^{1}$

Em geral, a estabilidade química do carbono (C) junto aos solos e sedimentos ocorre com a formação de substâncias húmicas, por meio de um processo genericamente denominado humificação. Este processo pode ser estimado pelo aumento da concentração de radicais livres do tipo semiquinonas (CRLS) nas SH, determinados por meio da espectroscopia de ressonância paramagnética eletrônica (RPE). ${ }^{2-10}$

Com o avanço da humificação ocorre a policondensação e a conjugação de estruturas insaturadas nas SH. Grupamentos bifenólicos de posição orto ou para, formados durante o processo de decomposição da matéria orgânica, são convertidos em quinonas, via reações de oxidação, as quais são precursoras de radicais livres do tipo semiquinonas nas $\mathrm{SH}$, que se estabilizam com o avanço da humificação. ${ }^{6-10}$ Adicionalmente, espécies de quinonas oxidadas e reduzidas participam da agregação das SH de forma estável, convergindo para a teoria dos arranjos supramoleculares dessas substâncias, possibilitando inferir que a atração entre quinonas doadoras e receptoras de elétrons possa ser computada entre as forças que mantêm tais associações. ${ }^{11}$

*e-mail: marihus@ufv.br
Os radicais livres do tipo semiquinonas das SH são grupos doadores de elétrons e, por meio de titulações redox iodimétricas, podem ser oxidados pelo iodo $\left(\mathrm{I}_{2}\right)$. As titulações redox permitem estimar o potencial formal padrão do eletrodo $\left({ }^{\mathrm{F}} \mathrm{E}_{\mathrm{H}}{ }^{\circ}\right)$ e a capacidade de oxidação (COx), fatores de intensidade e de capacidade da atividade de elétrons, respectivamente, análogos ao $\mathrm{pH}$ e a capacidade de neutralização ácida para o caso de prótons. ${ }^{1,3,12,13}$ Dessa forma, as titulações redox, analogamente à RPE, possibilitam estimar o grau de humificação das $\mathrm{SH}^{3-5}$

Trabalhos com amostras de diferentes ácidos húmicos, tituladas com um oxidante $\left(\mathrm{I}_{2}\right)$ em atmosfera inerte e condições especificadas, verificaram que os valores do ${ }^{\mathrm{F}} \mathrm{E}_{\mathrm{H}}{ }^{\mathrm{o}}$ foram semelhantes, entre 0,773 e $0,794 \mathrm{~V}$ a $25^{\circ} \mathrm{C} \cdot{ }^{2-5}$ A COx dos ácidos húmicos variou de 3,88 a 4,39 $\mathrm{mol}_{\mathrm{c}} \mathrm{kg}^{-1}$ a pH 5,0 e de 5,35 até 7,89 mol $_{\mathrm{c}} \mathrm{kg}^{-1}$ a pH 7,0. ${ }^{2-5}$ Foi observada correlação positiva e significativa entre a capacidade de oxidação dos ácidos húmicos e as suas concentrações de grupos funcionais fenólicos, quinonas e semiquinonas. ${ }^{3,4}$ Os autores observaram, ainda, que as reações químicas de derivação por metilação e acetilação das amostras de ácidos húmicos reduziram drasticamente, e de forma análoga, a COx dos ácidos húmicos, indicando a participação de grupos fenólicos e de quinonas reduzidas (e.g., semiquinonas e hidroquinonas) como grupos doadores de elétrons nessas substâncias.

As titulações redox da MO isolada de águas interiores e oceânicas podem fornecer informações qualitativas e quantitativas sobre as formas de carbono nesses sistemas naturais, sobretudo nas condições de oceanos profundos usados para a exploração petrolífera, tanto para conhecimento desta ambiência, como para o monitoramento de eventuais perturbações sob tal atividade. No ambiente oceânico, na interface entre a plataforma e a margem continental e no ambiente profundo, a relação entre fontes e imobilização de material particulado em suspensão e suas associações orgânicas e inorgânicas possui 
muitas incertezas, principalmente em relação aos mecanismos de preservação e transformação de $\mathrm{MO}$, nutrientes e metais nos sedimentos de fundo. A contaminação deste ambiente pode apresentar efeitos adversos nos ecossistemas aquáticos através do impacto nas estruturas das comunidades bentônicas. Assim, as titulações redox do material orgânico, gerando propriedades termodinâmicas, tais como as variáveis ${ }^{\mathrm{F}} \mathrm{E}_{\mathrm{H}}^{\mathrm{o}}, \mathrm{K}, \mathrm{pE}, \Delta \mathrm{G}^{\mathrm{o}}$ e $\mathrm{COx}$, possibilitam, por exemplo, verificar se a MO desses sistemas é semelhante à de outros compartimentos continentais, ou seja, permitindo avaliar a identidade química atual e monitorar eventuais efeitos de perturbações futuras nesses recursos naturais. Além disso, fornecem informações para a melhor compreensão da estabilidade dos estoques de $\mathrm{C}$ nos sistemas aquáticos e suas trocas entre as geosferas (e.g., pedosfera, biosfera, hidrosfera, atmosfera). Informações a respeito do estado redox da MO do ambiente de oceanos profundos e da sua tendência redox podem relacionar-se à especiação química em sistemas naturais. ${ }^{2,3,12-15}$ Elementos como $\mathrm{Fe}, \mathrm{Cu}, \mathrm{Hg}, \mathrm{V}$, entre outros, têm sido citados como participantes nas reações redox da MO nestes sistemas. ${ }^{16,17}$ Contudo, as informações sobre as propriedades redox da MO são escassas para os ambientes de oceanos profundos e poderão contribuir para o entendimento de mecanismos envolvendo a geoquímica, tanto de metais como de poluentes orgânicos em ambientes aquáticos, bem como as trocas de carbono no gradiente entre continente e oceano. ${ }^{18}$

O presente estudo objetivou o fracionamento da MO e o isolamento das $\mathrm{SH}$ de amostras de sedimentos coletadas em diferentes isóbatas $(750 ; 1.050 ; 1.350 ; 1.650 ; 1950 \mathrm{~m})$ de oceanos profundos, visando determinar suas propriedades redox usando titulações iodimétricas em atmosfera inerte e condições especificadas de $\mathrm{pH}$ e força iônica.

\section{PARTE EXPERIMENTAL}

\section{Ambiente em estudo e amostragem}

O estudo foi direcionado à Campanha Oceanográfica "Ocean Proof" da PETROBRAS (Petróleo do Brasil s/a) na Margem Continental da Bacia de Campos, Estado do Rio de Janeiro, sendo as latitudes e longitudes entre $22^{\circ} 12^{\prime}$ a $21^{\circ} 52^{\prime}$ e $39^{\circ} 40^{\prime}$ a $39^{\circ} 55^{\prime}$ para a área Norte e, variando de $22^{\circ} 10^{\prime}$ a $22^{\circ} 40^{\prime}$ e de $39^{\circ} 51^{\prime}$ a $40^{\circ} 21^{\prime}$ para a área Sul, respectivamente. O mapeamento da área e a amostragem foram realizados previamente e encontram-se detalhadamente descritos por Araújo et al. ${ }^{18}$

As isóbatas de amostragem $(750 \mathrm{~m}, 1.050 \mathrm{~m}, 1.350 \mathrm{~m}, 1.650$ $\mathrm{m}$ e $1.950 \mathrm{~m}$ ) foram selecionadas previamente pela PETROBRAS através do Projeto de Caracterização Ambiental de Águas Profundas da Bacia de Campos, coordenado pelo CENPES/PETROBRAS. A variabilidade espacial foi estudada em 2 eixos, o primeiro comparando a área Norte-Sul da Bacia de Campos e o segundo avaliando a variabilidade dos resultados em relação à profundidade das isóbatas. As áreas ao Norte e ao Sul foram formadas por 20 e 24 estações de coleta, respectivamente. As coletas foram realizadas a bordo do N/RB Astro Garoupa da PETROBRAS. Um box-corer da Ocean Instruments "Usnel Spade Corer", foi utilizado na amostragem das 44 estações das quais a fração de $0-2 \mathrm{~cm}$ de sedimentos superficiais foi a trabalhada neste estudo. Todas as amostras foram mantidas sob refrigeração no navio até o transporte para o laboratório. No laboratório, as amostras de sedimentos foram liofilizadas e seguiram para as análises. ${ }^{18}$

\section{Determinação da composição elementar e isotópica}

Inicialmente, cerca de 15 a $20 \mathrm{mg}$ das amostras de sedimentos foram acidificadas com $\mathrm{HCl} 1 \mathrm{~mol} \mathrm{~L}^{-1}$ diretamente em recipientes de prata para posterior determinação da composição elementar e isotópica. Este procedimento é necessário devido aos teores de carbonato (30 a 50\%) contidos nestes sedimentos da Margem Continental da Bacia de Campos. Após evaporação em estufa $\left(50{ }^{\circ} \mathrm{C}-48 \mathrm{~h}\right)$ as amostras foram fechadas e transferidas para o analisador elementar (Carlo Erba modelo EA $1110 \mathrm{CHN}$ ) acoplado a um espectrômetro de massa Finnigan Delta Plus. Os resultados da composição elementar da $\mathrm{MO}\left(\mathrm{C}_{\text {org }}\right.$ e $\left.\mathrm{N}_{\text {total }}\right)$ foram expressos em teores por massa de sedimento, enquanto as composições isotópicas de carbono e de nitrogênio $\left(\delta^{13} \mathrm{C}\right.$ e $\left.\delta^{15} \mathrm{~N}\right)$, consistem de valores relativos ao padrão PDB (Pee Dee Belemnite) e ao ar atmosférico, respectivamente. ${ }^{18}$ As análises foram realizadas no Laboratório de Ecologia Isotópica do Centro de Energia Aplicada à Agricultura (CENA - Universidade de São Paulo). ${ }^{18}$

\section{Fracionamento do carbono orgânico}

O fracionamento, a extração e a purificação do carbono orgânico dos sedimentos foi realizado conforme as recomendações da Sociedade Internacional de Substâncias Húmicas. ${ }^{19}$ Inicialmente as amostras foram tratadas com $\mathrm{HCl} 1 \mathrm{~mol} \mathrm{~L}^{-1}$ (agitação, em tubos de centrífuga, de $20 \mathrm{~g}$ de amostra: $200 \mathrm{~mL}$ de solução por 4 horas) para a solubilização de carbonatos e extração da fração de ácidos fúlvicos livres. Após tal agitação, foi realizada a centrifugação (Centrífuga HERMLE-Z382K) a $5.000 \mathrm{~g}$ por 30 minutos. A densidade ótica do extrato sobrenadante foi medida por espectrofotometria de absorção molecular em um equipamento Hitachi - UV-Vis 2000, nos comprimentos de onda de 250 e $465 \mathrm{~nm}$. Para a extração de substâncias orgânicas alcalino-solúveis o precipitado do extrato obtido na etapa anterior foi ajustado a $\mathrm{pH} 7 \mathrm{e}$, em seguida, adicionaram-se ao tubo de centrífuga $200 \mathrm{~mL}$ de $\mathrm{NaOH} 0,5 \mathrm{~mol} \mathrm{~L}^{-1}$ em atmosfera de $\mathrm{N}_{2}$. Tal sistema, a $\mathrm{pH}$ 13, foi agitado horizontalmente por 24 horas. As amostras foram centrifugadas e as densidades óticas dos sobrenadantes foram determinadas, bem como os teores de carbono orgânico desses sobrenadantes $\left(\mathrm{C}_{\mathrm{NaOH}}\right)$ e dos precipitados $\left(\mathrm{C}_{\mathrm{Residual}}\right)$. Parte dos sobrenadantes da operação anterior foi acidificada até $\mathrm{pH} 1,5 \mathrm{e}$, a seguir, centrifugada para a separação da $\mathrm{SH}$ em ácidos húmicos (precipitado) e ácidos fúlvicos (sobrenadante).

\section{Purificação da fração alcalino-solúvel}

Parte do extrato contendo a fração de $\mathrm{C}$ alcalino-solúvel foi acidificada a $\mathrm{pH} \sim 1,5$ e, a seguir, foi realizada a eluição em coluna contendo resina de retenção polar $\left(\mathrm{XAD}-8^{\circledR}\right)$ saturada positivamente e, na sequência, em coluna contendo resina trocadora de cátions Amberlite-IR $120^{\circledR} .{ }^{19}$

\section{Titulações redox}

As titulações redox consistiram da oxidação da $\mathrm{MO}$ com $\mathrm{I}_{2}$ (iodimetria), sob atmosfera inerte. ${ }^{2,3} \mathrm{O}$ sistema de titulação foi montado utilizando-se um frasco termostatizado e fechado com uma tampa adaptada para conter um eletrodo combinado de $\mathrm{pH}$ (pHmetro Quimis Q400A), um eletrodo redox combinado (Analyser 6A05-GK AgCl, $\mathrm{Ag} \| \mathrm{Pt}$ ), além de um tubo para entrada de gás argônio e uma microbureta de $2 \mathrm{~mL}$ (Gilmont ${ }^{\circledR}$ GS-1200-A, com subdivisões de $2 \mu \mathrm{L}$ ). $\mathrm{O}$ sistema foi termostatizado através de um banho em recirculação mantido a $25^{\circ} \mathrm{C}$ (Microquímica ${ }^{\circledR}$ Banho MQBCT 99-20).

No preparo do titulado, adicionaram-se ao frasco de titulação 125 $\mathrm{mL}$ de solução tampão fosfato $\left(\mathrm{Na}_{2} \mathrm{HPO}_{4} 0,05 \mathrm{~mol} \mathrm{~L}-1\right.$ e $\mathrm{NaH}_{2} \mathrm{PO}_{4}$. $\left.\mathrm{H}_{2} \mathrm{O} 0,05 \mathrm{~mol} \mathrm{~L}^{-1}\right)$ contendo $\mathrm{KCl} 0,05 \mathrm{~mol} \mathrm{~L}^{-1} \mathrm{e}$, em seguida, ajustou-se o pH com $\mathrm{HCl}$ ou $\mathrm{NaOH}$ para pH 5,00 ou 7,00. A solução foi agitada magneticamente e borbulhada com argônio por 60 minutos para a depleção de $\mathrm{O}_{2}$. Acrescentou-se MO à solução para obtenção 
de uma concentração em suspensão de $50 \mathrm{mg} \mathrm{L}^{-1}$. Novamente, agitou-se e borbulhou-se a suspensão com argônio durante 30 minutos. Em seguida, ajustou-se o pH da suspensão para 5 ou $7 \mathrm{com} \mathrm{HCl}$ ou $\mathrm{NaOH}$ para o início da titulação. ${ }^{2,3}$

Para cada titulação, calibrou-se o pHmetro utilizando soluções tampões a pH 4 e 7 . O eletrodo redox foi testado com solução tampão redox férrico-ferrosa, preparada com sulfato ferroso amoniacal 0,100 mol L ${ }^{-1}$, sulfato férrico amoniacal $0,100 \mathrm{~mol} \mathrm{~L}^{-1} \mathrm{e} \mathrm{H}_{2} \mathrm{SO}_{4} 1,000 \mathrm{~mol}$ $\mathrm{L}^{-1}$. A leitura da força eletromotriz da cela (f.e. $\mathrm{m}_{\text {cela }}$ ) em $439 \pm 2 \mathrm{mV}$ indicou o correto funcionamento do eletrodo. ${ }^{20}$ Padronizou-se a solução de $\mathrm{I}_{2}$ para cada titulação utilizando tiossulfato de sódio.

Nas titulações, utilizaram-se incrementos de $2 \mu \mathrm{L}$ de solução $0,025 \mathrm{~mol} \mathrm{~kg}^{-1}$ de $\mathrm{I}_{2}\left(\mathrm{~mol} \mathrm{~kg} \mathrm{~kg}^{-1}=\right.$ molalidade, ou seja, o número de mols de $\mathrm{I}_{2}$ por massa de solução), ${ }^{2-6}$ com densidade conhecida ( $\mathrm{kg}$ de $\mathrm{I}_{2}$ por kg de solução) e, após cada incremento de titulante, a solução foi agitada e borbulhada com argônio por mais 30 segundos para facilitar a homogeneização do sistema. Em seguida, registrou-se a leitura da f.e.m. cela após cada adição de titulante, sempre quando a variação no

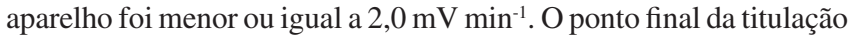
redox de ácidos húmicos foi atingido quando valores constantes de

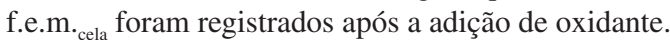

As curvas de titulação redox foram obtidas relacionando-se num

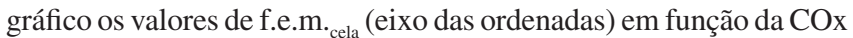
da MO (eixo das abscissas). O valor da COx foi determinado pelo número de mols de $\mathrm{I}_{2(\mathrm{aq})}$ reduzidos a $\mathrm{I}_{(\mathrm{aq})}^{-}$na reação por unidade de massa de material orgânico na fração alcalino solúvel e por massa de carbono na MO $\left(\mathrm{mol}_{\mathrm{c}} \mathrm{kg}^{-1}\right)$. Determinou-se o ponto de inflexão de cada curva de titulação redox obtendo-se a segunda derivada da

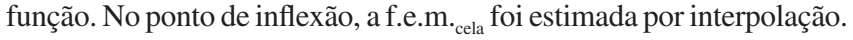
Adicionalmente, realizaram-se titulações de amostras em branco, sem adição de MO. . $^{2-6}$

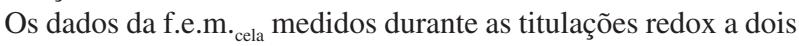
valores de $\mathrm{pH}$ ( 5 e 7), foram usados para determinar o consumo de prótons e de elétrons, $\mathrm{O}{ }^{\mathrm{F}} \mathrm{E}_{\mathrm{H}}{ }^{0}$, a variação de energia livre padrão de Gibbs $\left(\Delta \mathrm{G}^{\circ}\right)$, a constante condicional de equilíbrio $\left(\mathrm{K}_{c}\right)$ e o inverso do logarítimo da atividade de elétrons $(\mathrm{pE})$ para a semirreação da MO escrita como reação de redução por convenção da Sociedade Internacional de Química Pura e Aplicada (IUPAC), visando facilitar a comparação de pares redox pelos potenciais de redução:-2-6

$$
\mathrm{MO}_{\mathrm{Ox}}+\mathrm{ne}^{-}+\mathrm{qH}^{+}=\mathrm{MO}_{\mathrm{Red}}
$$

\section{Análises estatísticas}

Estimou-se o desvio-padrão da média para cada variável experimental e, também, a matriz de correlações lineares de Pearson para as variáveis estudadas. As estimativas dos coeficientes de correlação foram submetidas ao teste $\mathrm{F}$, aos níveis de 1,5 e $10 \%$ de probabilidade. ${ }^{21}$

\section{RESULTADOS E DISCUSSÃO}

O intervalo de concentração do $\mathrm{C}_{\text {org }}$ nos sedimentos de Norte e Sul foi de 12,3 a 17,4 $\mathrm{g} \mathrm{kg}^{-1}$ e de 12,9 a 17,4 $\mathrm{g} \mathrm{kg}^{-1}$, respectivamente, sendo observada uma baixa variabilidade entre as estações (Tabela 1). A concentração de $\mathrm{N}_{\text {Total }}$, embora tenha apresentado um menor intervalo, 1,4 a 2,1 $\mathrm{g} \mathrm{kg}^{-1}$ e 1,5 a 2,1 $\mathrm{g} \mathrm{kg}^{-1}$, em amostragens ao Norte e ao Sul respectivamente, mostrou variabilidade análoga à observada para o $\mathrm{C}_{\text {org }}$

As concentrações de $\mathrm{C}_{\text {org }}$ que pouco variaram com as direções amostradas aumentaram em função da profundidade estudada (Figura 1). Em ambos os casos, os teores máximos de concentração foram observados nas amostras coletadas em maior profundidade. Araújo et al. ${ }^{18}$ também verificaram baixa variabilidade de granulometria dos sedimentos e teores de $\mathrm{Hg}$ nessas mesmas condições.

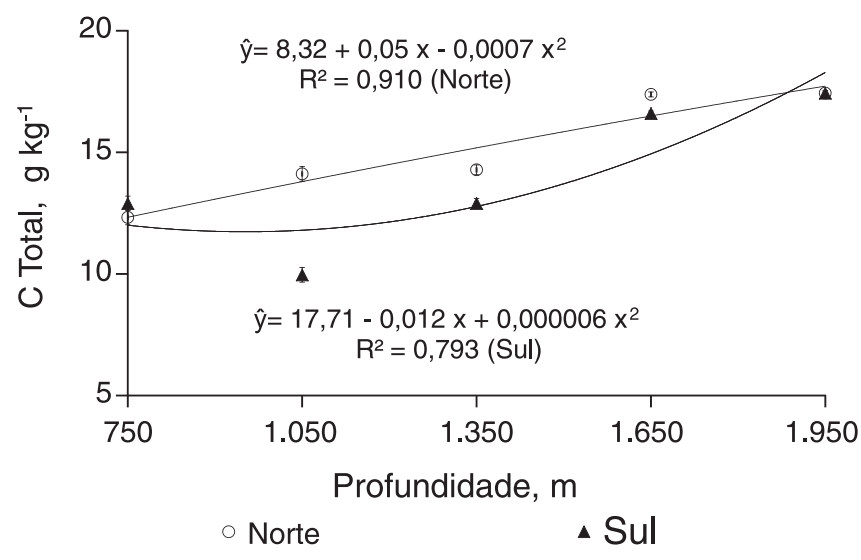

Figura 1. Teores de carbono orgânico total dos sedimentos em oceanos profundos da bacia petrolífera Norte Fluminense em função da profundidade de amostragem

Examinando os demais dados apresentados na Tabela 1 pode-se constatar a homogeneidade espacial nos dados de $\delta^{13} \mathrm{C}$ e $\delta^{15} \mathrm{~N}$ e relação C:N no eixo Norte - Sul e, portanto, infere-se que a fonte da MO é comum nas duas áreas. Em relação à variabilidade ao longo do gradiente de profundidade temos que a área Norte apresentou baixa variabilidade nas concentrações de $\mathrm{C}_{\text {org }}$ e $\mathrm{N}_{\text {Total }}$, assim como para composição isotópica $\left(\delta^{13} \mathrm{C}\right.$ e $\left.\delta^{15} \mathrm{~N}\right)$ e razão $\mathrm{C}: \mathrm{N}$. Somente o $\delta^{15} \mathrm{~N}$ incrementou em direção às áreas mais profundas e este padrão pode ser decorrente da geoquímica recente, que libera para a água de contato ou intersticial o isótopo mais leve do nitrogênio. Já a composição isotópica do $\mathrm{C}_{\mathrm{org}}$ é tipicamente fitoplanctônica de origem marinha e os dados sedimentares expressam a contribuição desta fonte na formação dos depósitos de material orgânico estudados.

Kao et al. $^{22}$ também encontraram os valores mais positivos de

Tabela 1. Teores de nitrogênio carbono e nitrogênio isotópicos $\left(\delta^{15} \mathrm{~N}\right.$ e $\left.\delta^{13} \mathrm{C}\right)$, de carbono e nitrogênio totais $\left(\mathrm{C}_{\text {Total }}\right.$ e $\left.\mathrm{N}_{\text {Total }}\right)$ e relação C:N da matéria orgânica nos sedimentos ${ }^{(1)}$

\begin{tabular}{|c|c|c|c|c|c|c|c|c|c|c|}
\hline \multirow{2}{*}{ Isóbata } & \multicolumn{5}{|c|}{ Norte } & \multicolumn{5}{|c|}{ Sul } \\
\hline & $\delta^{15} \mathrm{~N}$ & $\delta^{13} \mathrm{C}$ & $\mathrm{C}_{\text {Total }}$ & $\mathrm{N}_{\text {Total }}$ & $\mathrm{C}: \mathrm{N}$ & $\delta^{15} \mathrm{~N}$ & $\delta^{13} \mathrm{C}$ & $\mathrm{C}_{\text {Total }}$ & $\mathrm{N}_{\text {Total }}$ & $\mathrm{C}: \mathrm{N}$ \\
\hline $\mathrm{m}$ & \multicolumn{5}{|c|}{ 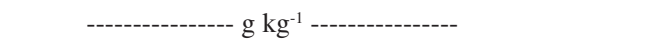 } & \multicolumn{5}{|c|}{ 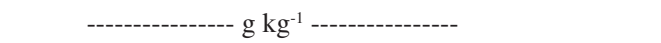 } \\
\hline 750 & $5,7 \pm 0,4$ & $-21,7 \pm 0,3$ & $12,3 \pm 0,4$ & $1,4 \pm 0,3$ & 8,8 & $5,7 \pm 0,5$ & $-21,9 \pm 0,4$ & $12,9 \pm 0,3$ & $1,5 \pm 0,4$ & 8,6 \\
\hline 1.050 & $6,5 \pm 0,5$ & $-21,5 \pm 0,3$ & $14,1 \pm 0,2$ & $1,7 \pm 0,4$ & 8,3 & $6,8 \pm 0,6$ & $-22,2 \pm 0,5$ & $10,0 \pm 0,3$ & $1,2 \pm 0,3$ & 8,3 \\
\hline 1.350 & $6,4 \pm 0,3$ & $-21,5 \pm 0,2$ & $14,3 \pm 0,4$ & $1,7 \pm 0,3$ & 8,4 & $6,9 \pm 0,3$ & $-21,6 \pm 0,3$ & $12,9 \pm 0,2$ & $1,5 \pm 0,3$ & 8,6 \\
\hline 1.650 & $7,6 \pm 0,4$ & $-21,0 \pm 0,1$ & $17,4 \pm 0,3$ & $2,2 \pm 0,1$ & 7,9 & $7,4 \pm 0,3$ & $-21,1 \pm 0,2$ & $16,6 \pm 0,1$ & $2,0 \pm 0,1$ & 8,3 \\
\hline 1.950 & $7,3 \pm 0,4$ & $-20,8 \pm 0,1$ & $17,4 \pm 0,1$ & $2,1 \pm 0,1$ & 8,3 & $7,1 \pm 0,5$ & $-20,9 \pm 0,1$ & $17,4 \pm 0,1$ & $2,1 \pm 0,1$ & 8,3 \\
\hline
\end{tabular}

${ }^{(1)}$ As médias experimentais estão acompanhadas pelo seu desvio-padrão. 
Tabela 2. Formas ${ }^{(1)}$ de carbono (C) nas amostras de sedimentos coletados em oceano profundo

\begin{tabular}{|c|c|c|c|c|c|c|c|c|}
\hline \multirow{3}{*}{$\begin{array}{c}\text { Isóbata } \\
\mathrm{m}\end{array}$} & \multicolumn{4}{|c|}{ Norte } & \multicolumn{4}{|c|}{ Sul } \\
\hline & \multicolumn{2}{|c|}{$\mathrm{C}_{\mathrm{NaOH}}$} & \multicolumn{2}{|c|}{$\mathrm{C}_{\text {Residual }}$} & \multicolumn{2}{|c|}{$\mathrm{C}_{\mathrm{NaOH}}$} & \multicolumn{2}{|c|}{$\mathrm{C}_{\text {Residual }}$} \\
\hline & $\mathrm{g} \mathrm{kg}^{-1}$ & $\%$ do $C_{\mathrm{T}}$ & $\mathrm{g} \mathrm{kg}^{-1}$ & $\%$ do $C_{\mathrm{T}}$ & $\mathrm{g} \mathrm{kg}^{-1}$ & $\%$ do $\mathrm{C}_{\mathrm{T}}$ & $\mathrm{g} \mathrm{kg}^{-1}$ & $\%$ do $C_{\mathrm{T}}$ \\
\hline 750 & $0,75 \pm 0,03$ & 4 & $11,5 \pm 0,6$ & 93 & $0,47 \pm 0,02$ & 6 & $11,8 \pm 0,4$ & 91 \\
\hline 1.050 & $0,86 \pm 0,02$ & 3 & $13,5 \pm 0,4$ & 96 & $0,40 \pm 0,02$ & 9 & $9,2 \pm 0,5$ & 92 \\
\hline 1.350 & $0,48 \pm 0,04$ & 5 & $13,0 \pm 0,3$ & 91 & $0,66 \pm 0,04$ & 4 & $12,7 \pm 0,3$ & 98 \\
\hline 1.650 & $1,30 \pm 0,03$ & 5 & $16,0 \pm 0,5$ & 92 & $0,93 \pm 0,03$ & 8 & $15,1 \pm 0,5$ & 91 \\
\hline 1.950 & $1,21 \pm 0,02$ & 4 & $16,6 \pm 0,4$ & 95 & $0,61 \pm 0,02$ & 7 & $16,7 \pm 0,6$ & 96 \\
\hline
\end{tabular}

${ }^{(1)} \mathrm{C}_{\mathrm{NaOH}}$ e $\mathrm{C}_{\text {Residual: }}$ frações obtidas no sobrenadante e no precipitado, respectivamente, após a centrifugação dos extratos obtidos a partir de amostras de sedimentos agitadas com $\mathrm{NaOH} 0,5 \mathrm{~mol} \mathrm{~L}^{-1}$ por 16 horas na relação sedimento: extrator igual a 20:1; As médias experimentais encontram-se acompanhadas de seu desvio-padrão.

$\delta^{13} \mathrm{C}$ nas estações mais profundas $(\approx 2.000 \mathrm{~m})$ na região de Taiwan e associaram esses valores a fontes marinhas de MO. Cabe ressaltar que neste estudo as estações mais rasas foram coletadas na isóbata de 30 metros, ao contrário do presente trabalho, em que todas as estações são localizadas em áreas com profundidades superiores a $700 \mathrm{~m}$.

Os resultados possibilitam inferir que o aporte de MO seja semelhante em toda a área de estudo e, também, a produtividade primária. Porém, o maior tempo de residência das partículas na coluna d'água tendeu a favorecer o processo de decomposição da MO, até sua chegada nos sedimentos. A combinação entre estes processos acrescidos do metabolismo bentônico, conforme observado para os resultados do $\delta^{15} \mathrm{~N}$, caracteriza o padrão de fonte comum atuando na região, sendo que existe a tendência de variação do material orgânico depositado ao longo das isóbatas.

\section{Fracionamento químico da matéria orgânica}

Para os extratos obtidos pela agitação das amostras em $\mathrm{HCl}$ $1 \mathrm{~mol} \mathrm{~L}^{-1}$ a densidade ótica, medida em UV-Vis em 250 e $465 \mathrm{~nm}$, não diferiu daquela apresentada pela prova em branco, sem a adição de sedimentos. Tal fato mostra que nos sedimentos de oceanos profundos amostrados, para o presente estudo, não foi possível computar a participação significativa da fração ácidos fúlvicos livres.

Em termos de quantidade de carbono orgânico total, não há diferenças espaciais significativas no eixo norte - sul (Tabela 1). Contudo, houve indicação de alteração nos compartimentos da MO (Tabela 2).

O fracionamento químico da MO dos sedimentos (Tabela 2) mostrou que a fração residual $\left(\mathrm{C}_{\text {Residual }}\right)$ constitui, em média, 94 e 93 $\%$ do carbono orgânico total nas amostras nas direções norte e sul, respectivamente. Quanto às substâncias orgânicas extraídas com $\mathrm{NaOH} 0,5 \mathrm{~mol} \mathrm{~L}^{-1}$, após a acidificação desse extrato a pH 1, não foi detectada a precipitação de ácidos húmicos no sistema, indicando que o material orgânico encontrado nessa fração alcalino-solúvel $\left(\mathrm{C}_{\mathrm{NaOH}}\right)$ foi composto apenas pela fração operacionalmente denominada ácidos fúlvicos.

À semelhança dos resultados da concentração de $\mathrm{C}_{\mathrm{Total}}$ (Figura 1), os valores observados para o $C_{\text {Residual }}$ também apresentaram-se incrementados com o aumento da profundidade (Figura 2). As taxas de incremento também se assemelharam àquelas determinadas para o $\mathrm{C}_{\text {Total }}$ e os pontos de máxima das curvas foram aqueles das maiores isóbatas.

Para os valores de $\mathrm{C}_{\mathrm{NaOH}}$ (Figura 3), fração pela qual é esperada a reatividade característica das $\mathrm{SH}$, maiores teores foram observados para as amostras coletadas ao norte do que para aquelas coletadas ao sul, cujos valores, em média, foram de 0,92 a $0,61 \mathrm{~g} \mathrm{~kg}^{-1}$, respectivamente. Em relação ao $\mathrm{C}_{\text {Total }}$, porém, observou-se uma participação de 4 e de $7 \%$ para as amostras ao norte e ao sul, respectivamente. Os

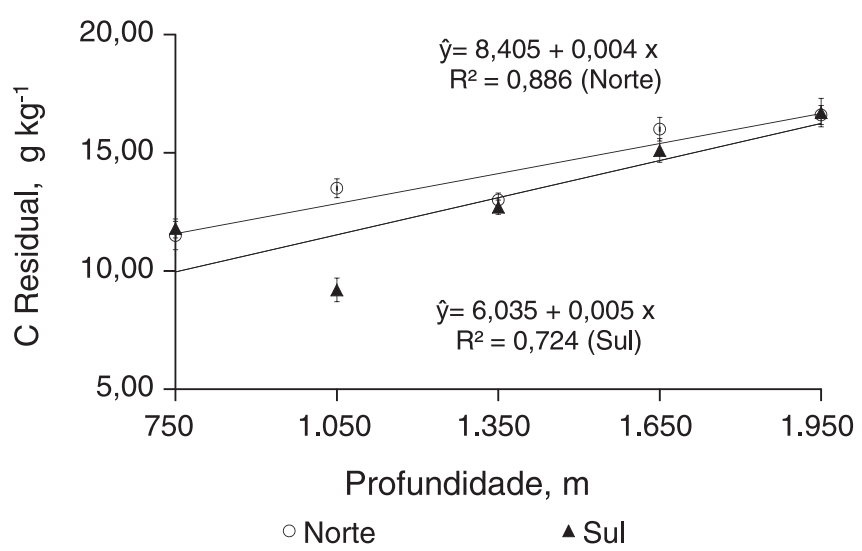

Figura 2. Teores de carbono orgânico residual $\left(C_{\text {Residual }}\right)$ dos sedimentos em oceanos profundos da bacia petrolífera Norte Fluminense em função da profundidade de amostragem

teores de $\mathrm{C}_{\mathrm{NaOH}}$ tenderam, mesmo que não de forma generalizada, a aumentar com a profundidade, mostrando os maiores valores na isóbata de $1.650 \mathrm{~m}$ e ligeira tendência à diminuição na isóbata $1.950 \mathrm{~m}$, mas ainda superior aqueles determinados à menores profundidades.

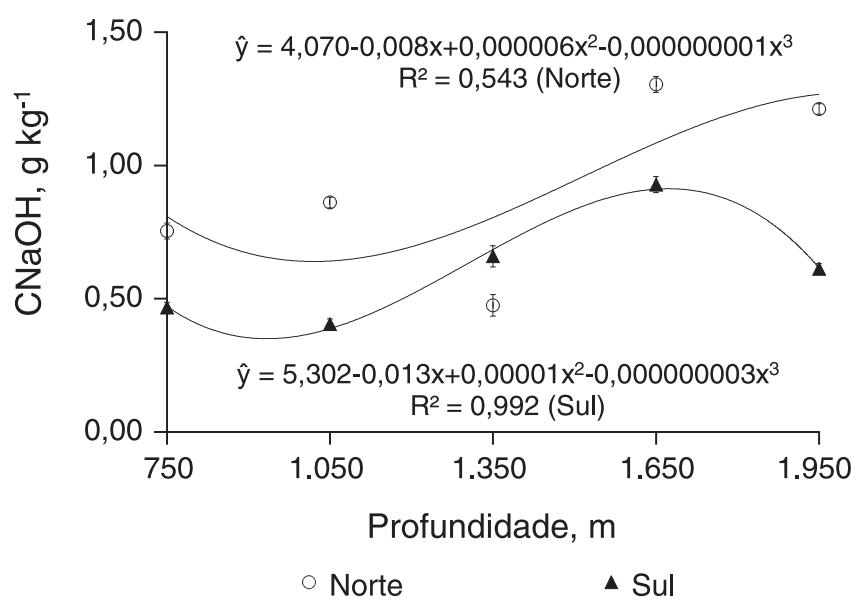

Figura 3. Teores de carbono orgânico alcalino-solúvel $\left(C_{\mathrm{NaOH}}\right)$ dos sedimentos em oceanos profundos da bacia petrolífera Norte Fluminense em função da profundidade de amostragem

\section{Titulações redox}

Duas das curvas de titulação redox iodimétrica encontram-se na Figura 4, onde estão apresentadas a f.e.m. cela $_{\text {em }} \mathrm{mV}$ e a $\mathrm{COx}$ por 
unidade de massa de carbono $\left(\mathrm{mol}_{\mathrm{c}} \mathrm{kg}^{-1}\right)$ a partir de seus teores nos sedimentos, a pH 5,0.

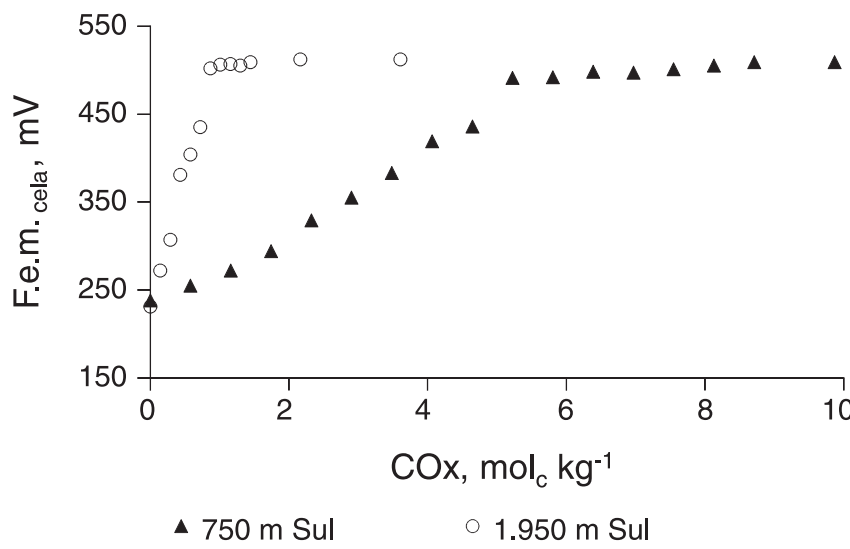

Figura 4. Titulações redox da matéria orgânica alcalino-solúvel isolada dos sedimentos em oceanos profundos da bacia petrolifera Norte Fluminense, em solução tampão de fosfatos $0,05 \mathrm{~mol} \mathrm{~L}^{-1}$, a pH 5,00, contendo $\mathrm{KCl}$ 0,05 $\mathrm{mol} \mathrm{L}^{-1}$. Dados: fem $m_{\text {cela }}=465$ e $467 \mathrm{mV}$ e COx $=4,64$ e 0,72 $\mathrm{mol}_{\mathrm{c}} \mathrm{kg}^{-1}$ (corrigido por massa de $C$ a partir de sua concentração nos sedimentos), para as amostras coletadas ao sul nas isóbatas 750 e $1.950 \mathrm{~m}$, respectivamente

Os valores de f.e.m. cela $_{\text {, }}$ para as titulações redox realizadas a $\mathrm{pH} 5$ e pH 7, e os resultados dos cálculos de ${ }^{\mathrm{F}} \mathrm{E}_{\mathrm{H}}{ }^{\mathrm{o}}, \log \mathrm{K}_{\mathrm{c}}, \mathrm{pE}_{\mathrm{c}}$ encontram-se apresentados no Tabela 3 . As curvas de titulação potenciométrica da MO isolada dos sedimentos de oceanos profundos foram semelhantes às obtidas por Struyk e Sposito, ${ }^{2}$ usando três amostras-padrão de ácidos húmicos da IHSS, e às apresentadas por Baldotto et al. ${ }^{3-6}$, usando amostras de ácidos húmicos isoladas de adubos orgânicos, solos sob diferentes manejos, águas interiores e estuarinas. Observa-se que, mesmo trabalhando com materiais orgânicos de diferentes origens, as propriedades redox apresentaram baixa variação, indicando que são atributos intrínsecos às substâncias húmicas.

No presente estudo os valores de ${ }^{\mathrm{F}} \mathrm{E}_{\mathrm{H}}{ }^{\mathrm{o}}$ para a semirreação de redução da $\mathrm{MO}$ isolada dos sedimentos de oceanos profundos pouco variaram, assumindo valores de 0,760 a 0,798 V. Struyk e Sposito ${ }^{2}$ encontraram valores de ${ }^{\mathrm{F}} \mathrm{E}_{\mathrm{H}}{ }^{\mathrm{o}}$ próximos a $0,780 \mathrm{~V}$ e Baldotto et al. ${ }^{3-6}$ obtiveram valores em torno de $0,782 \mathrm{~V}$. Em todos os trabalhos foram usadas soluções aquosas de $\mathrm{I}_{2}$ como oxidante. De forma semelhante, as demais propriedades redox da MO estimadas a partir do potencial redox $\left(\mathrm{K}_{\mathrm{c}}, \mathrm{pE}_{\mathrm{c}}\right.$ e $\left.\Delta \mathrm{G}^{\mathrm{o}}\right)$ também não se alteram com a origem das amostras. Contudo, essas propriedades puderam caracterizar o par redox $\mathrm{MO}_{\mathrm{Ox}}, \mathrm{MO}_{\mathrm{Red}} \mathrm{e}$, nesse sentido, pode-se inferir que a reação de redução (Eq. 1) é termodinamicamente favorecida nas condições de sistemas naturais como os sedimentos aqui estudados (valores negativos de $\Delta \mathrm{G}^{\circ}$ ). Além disso, a $\mathrm{MO}$ dos oceanos profundos apresenta ${ }^{\mathrm{F}} \mathrm{E}_{\mathrm{H}}{ }^{\circ}$, $\mathrm{pE}_{\mathrm{c},} \log \mathrm{K}_{\mathrm{c}}$ e de $\Delta \mathrm{G}^{\mathrm{o}}$ análogos aos de quinonas. ${ }^{14,15}$ No entanto, essas substâncias diferenciaram-se pela mais ampla relação entre prótons e elétrons transferidos na reação verificada para os grupos quinona $(1,00)$ em relação à MO do presente estudo $(0,33) \cdot{ }^{14,15}$

Uma comparação entre os valores de alguns pares redox comuns nos sistemas naturais permitiu alocar o par redox $\mathrm{MO}_{\mathrm{Ox}}, \mathrm{MO}_{\mathrm{Red}}$ numa "seqüência redox". ${ }^{2}$ Tal procedimento foi também realizado para os valores observados para as amostras do presente estudo e, dada a semelhança entre os valores das suas propriedades redox com as dos estudos supracitados, podem ser obtidas as mesmas inferências quanto à sua reatividade nos sistemas naturais. Assim, termodinamicamente, os ácidos húmicos podem reduzir $\mathrm{Hg}(\mathrm{II})$ e $\mathrm{Mn}(\mathrm{IV})$. Tais reduções foram observadas experimentalmente incubando tais íons metálicos com bactérias redutoras e ácidos húmicos. ${ }^{23-26}$

Para a predição da atividade das espécies oxidada e reduzida de $\mathrm{MO}\left(\mathrm{MO}_{\mathrm{Ox}, \text { Red }}\right)$ nos sistemas naturais é possível posicionar o par redox, com os dados médios das amostras estudadas, em diagramas $\mathrm{pE}$ vs. $\mathrm{pH} \cdot{ }^{3,23-26} \mathrm{O}$ material orgânico aqui estudado poderia, também, atuar como doador de elétrons nas reações redox em tais sistemas. Indica-se que grupos funcionais presentes na MO de sistemas naturais poderiam mediar a redução microbiológica de $\mathrm{Fe}(\mathrm{III})$ a partir da fase sólida, como por exemplo a espécie FeOOH. ${ }^{16,17}$ Contudo, se a redução de $\mathrm{Fe}(\mathrm{III})$ a partir da fase sólida acontece, deve, então, haver uma entrada externa de energia, pois tal reação ocorreria contra a tendência termodinâmica. Assim, naqueles estudos após a redução microbiológica, os grupos funcionais doadores possivelmente apresentaram um potencial abaixo daqueles determinados para as $\mathrm{SH}$ na ausência de microrganismos.

\section{Capacidade de oxidação}

Os valores da COx por massa de carbono da $\mathrm{MO}$ dos sedimentos de oceanos profundos (Tabela 4) variaram de 1,00 a 5,16 e de 1,72 a 7,97 mmol $_{\mathrm{c}} \mathrm{kg}^{-1}$ a pH 5 e de 0,65 a 4,94 e de 1,01 a 7,26 mmol $\mathrm{kg}^{-1}$, a pH 7,0, para as coletas ao norte a ao sul, respectivamente. De forma análoga às demais variáveis, a $\mathrm{COx}$ da $\mathrm{MO}$ dos sedimentos dos oceanos profundos também apresentou baixa variação entre as

Tabela 3. Propriedades redox ${ }^{(1)}$ da matéria orgânica isolada dos sedimentos em oceanos profundos da bacia petrolífera Norte Fluminense

\begin{tabular}{|c|c|c|c|c|c|c|c|c|}
\hline \multirow{3}{*}{ Amostra } & \multicolumn{2}{|c|}{ f.e.m $m_{\text {cela }}$} & \multirow{3}{*}{$\mathrm{q} / \mathrm{n}$} & \multirow{3}{*}{$\frac{{ }^{\mathrm{F}} \mathrm{E}_{\mathrm{H}}^{\circ}}{\mathrm{V}}$} & \multirow{3}{*}{$\log \mathrm{K}_{\mathrm{c}}$} & \multicolumn{2}{|c|}{$\mathrm{pE}_{\mathrm{c}}$} & \multirow{2}{*}{$\Delta \mathrm{G}^{\circ}$} \\
\hline & 5 & 7 & & & & 5 & 7 & \\
\hline & \multicolumn{2}{|c|}{$\mathrm{mV}$} & & & & & & cal mol-1 \\
\hline 750 Norte & $473 \pm 2$ & $441 \pm 1$ & 0,27 & 0,775 & 13,1 & 11,7 & 11,2 & $-17,9$ \\
\hline 1.050 Norte & $475 \pm 5$ & $446 \pm 3$ & 0,24 & 0,769 & 13,0 & 11,8 & 11,3 & $-17,7$ \\
\hline 1.350 Norte & $475 \pm 4$ & $440 \pm 5$ & 0,30 & 0,785 & 13,3 & 11,8 & 11,2 & $-18,1$ \\
\hline 1.650 Norte & $465 \pm 3$ & $424 \pm 2$ & 0,34 & 0,788 & 13,1 & 11,6 & 10,9 & $-18,2$ \\
\hline 1.950 Norte & $463 \pm 4$ & $430 \pm 8$ & 0,28 & 0,766 & 13,2 & 11,6 & 11,0 & $-17,7$ \\
\hline $750 \mathrm{Sul}$ & $466 \pm 2$ & $422 \pm 2$ & 0,37 & 0,796 & 13,4 & 11,6 & 10,9 & $-18,3$ \\
\hline $1.050 \mathrm{Sul}$ & $469 \pm 5$ & $431 \pm 7$ & 0,32 & 0,786 & 13,3 & 11,7 & 11,0 & $-18,1$ \\
\hline $1.350 \mathrm{Sul}$ & $467 \pm 3$ & $429 \pm 3$ & 0,32 & 0,784 & 13,4 & 11,6 & 11,0 & $-18,1$ \\
\hline $1.650 \mathrm{Sul}$ & $466 \pm 2$ & $428 \pm 3$ & 0,32 & 0,783 & 13,2 & 11,6 & 11,0 & $-18,1$ \\
\hline $1.950 \mathrm{Sul}$ & $467 \pm 2$ & $426 \pm 2$ & 0,34 & 0,791 & 13,2 & 11,6 & 11,0 & $-18,2$ \\
\hline
\end{tabular}

(1) Propriedades redox: definidas na seção Material e Métodos. As médias experimentais estão acompanhadas pelo seu desvio-padrão. 
direções de amostragem norte e sul. Fatores como morfologia do sedimento (ex: turbiditos e bioturbação) e diferenças no relevo entre as duas regiões não influenciaram as propriedades termodinâmicas da MO, mesmo sendo o ambiente de margem continental com presença de vários cânions (região norte) e platôs (região sul) que apresentam diferenças abruptas de declividade, gerando uma maior instabilidade para a região norte, onde uma migração pós-deposicional de sedimento tende a ocorrer com maior frequência. ${ }^{18}$

Tabela 4. Capacidade de oxidação( ${ }^{(1)}$ da matéria orgânica dos sedimentos

\begin{tabular}{ccc}
\hline & \multicolumn{2}{c}{$\mathrm{pH}$} \\
\cline { 2 - 3 } Amostra & \multicolumn{2}{c}{5} \\
\cline { 2 - 3 } & $5,16 \pm 0,47$ & $7,97 \pm 0,40$ \\
\hline 750 Norte & $4,54 \pm 0,22$ & $5,84 \pm 0,25$ \\
1.050 Norte & $1,46 \pm 0,13$ & $2,26 \pm 0,10$ \\
1.350 Norte & $1,23 \pm 0,09$ & $1,79 \pm 0,09$ \\
1.650 Norte & $1,00 \pm 0,14$ & $1,72 \pm 0,05$ \\
1.950 Norte & $4,94 \pm 0,29$ & $7,26 \pm 0,30$ \\
\hline 750 Sul & $3,30 \pm 0,25$ & $4,83 \pm 0,25$ \\
1.050 Sul & $1,29 \pm 0,18$ & $2,39 \pm 0,19$ \\
1.350 Sul & $0,74 \pm 0,07$ & $1,07 \pm 0,09$ \\
1.650 Sul & $0,65 \pm 0,07$ & $1,01 \pm 0,17$ \\
1.950 Sul &
\end{tabular}

(1) As médias experimentais estão acompanhadas pelo seu desvio-padrão.

A COx determinada para ácidos húmicos isolados de amostras-padrão da IHSS variou entre 1,09 e $6,50 \mathrm{~mol}_{\mathrm{c}} \mathrm{kg}^{-1} \mathrm{a} \mathrm{pH} 5$ e variando de 3,30 até 11,50 a pH 7. ${ }^{2}$ Ácidos húmicos obtidos de adubos orgânicos apresentaram COx de 3,88 a 4,39 a pH 5 e de 5,35 a 7,89 a pH 7. ${ }^{3}$ Observa-se, portanto, a variação da $\mathrm{COx}$ com o pH da titulação e com a origem das amostras. No primeiro caso, tal variação é devida ao consumo prótons nas reações redox de materiais orgânicos em sistemas naturais, resultando em incrementos da COx com o aumento do $\mathrm{pH}$. Tais variações da $\mathrm{COx}$ com o $\mathrm{pH}$ podem ser entendidas de acordo com a equação 1 . Já a variação da COx com a origem das amostras é resultado da diferença químico-estrutural das amostras, tendo sido observados aumentos da COx com a aromaticidade da MO. Essa aromaticidade correlaciona-se com a concentração de radicais livres tipo semiquinonas nos materiais orgânicos, admitidos como principais grupos doadores de elétrons nas condições especificadas para a titulação redox citadas. ${ }^{2,3}$

No presente estudo, em média, a quantidade de elétrons transferidos durante a titulação redox da MO estudada, por massa de carbono, foi de 2,68 e 2,18 mol $_{\mathrm{c}} \mathrm{kg}^{-1}$ a pH 5 e de 3,31 e 3,92 mol $_{\mathrm{c}} \mathrm{kg}^{-1}$ a pH 7, para as amostras coletadas ao norte e ao sul, respectivamente, resultando em incrementos de $\mathrm{COx}$ de aproximadamente $23 \%$ por unidade de $\mathrm{pH}$, dentro do intervalo de $\mathrm{pH}(\mathrm{pH} 5$ e $\mathrm{pH} 7$ ) aqui estudado. Struyk e Sposito ${ }^{2}$ e Baldotto et al. ${ }^{3}$ observaram que esse aumento foi de cerca de 35 e $32 \%$, respectivamente, no mesmo intervalo de $\mathrm{pH}$.

A COx do material orgânico isolado na fração alcalino solúvel no presente trabalho, representada apenas pelos ácidos fúlvicos, é coerente com os menores teores de grupos funcionais do tipo quinonas presentes nessas substâncias em comparação com os ácidos húmicos. Dobbss et al. ${ }^{27}$ estudando Latossolos de diferentes regiões brasileiras, verificaram que a $\mathrm{COx}$ em $\mathrm{pH} 7$ variou positivamente com o grau de hidrofobicidade (relação ácidos húmicos/ácidos fúlvicos) e de aromaticidade (teor de semiquinonas e de fenóis; e espectroscopias de infravermelho, fluorescência, ressonância magnética e UV-Visível), sendo as médias da $\mathrm{COx}$, de 2,50 $\mathrm{mol}_{\mathrm{c}} \mathrm{kg}^{-1}$ para os ácidos húmicos e de $1,74 \mathrm{~mol}_{\mathrm{c}} \mathrm{kg}^{-1}$ para os ácidos fúlvicos, condizentes com os aspectos químico-estruturais dessas substâncias, ou seja, diferentes aromaticidade ou hidrofobicidade.

Baldotto et al. ${ }^{5}$ determinaram a COx da MO obtida por ultrafiltração das águas superficiais num mesmo local do Rio Paraíba do Sul e verificaram variações com o $\mathrm{pH}$ do sistema e com a estação do ano. Para comparação, Baldotto et al. ${ }^{6}$ estudaram os sedimentos de fundo no mesmo local onde foram amostradas as águas superficiais e ao longo do gradiente fluvial-estuarino da bacia hidrográfica. A COx observada para tais materiais, por massa de carbono no material ultrafiltrado, foi de 0,48 a 0,85 mol $_{\mathrm{c}} \mathrm{kg}^{-1}$ a pH 5,0 e de 0,76 a 1,19 $\mathrm{mol}_{\mathrm{c}} \mathrm{kg}^{-1}$ a $\mathrm{pH} 7,0$, nas águas superficiais e, para os sedimentos de fundo, variando de 4,36 a 9,25 a pH 7,0. A MO isolada do material ultrafiltrado do Rio Paraíba do Sul apresentou modificações em sua atividade redox de acordo com a época de amostragem. Os autores inferiram sobre a composição de materiais em suspensão transportados na coluna d'água durante as estações do ano. Já os sedimentos de fundo revelaram que a MO humificada é mais estável nas porções fluviais do que nas estuarinas. Em conjunto, os resultados revelam a tendência de que os materiais em suspensão (mais solúveis, menos hidrofóbicos e aromáticos) apresentam menor COx que aqueles encontrados em sedimentos de fundo. Proporcionalmente, a COx dos sedimentos em suspensão é diluída pelo enriquecimento em ácidos fúlvicos. No presente trabalho, as substâncias alcalino solúveis isoladas dos sedimentos em oceanos profundos não contiveram o compartimento mais hidrofóbico, ou seja, os ácidos húmicos, o que converge para a menor COx determinada, indicativa de substâncias menos hidrofóbicas ou aromáticas.

Se, como já apresentado, para os teores das frações de C orgânico estudadas neste trabalho, observaram-se incrementos com o aumento da profundidade de amostragem (Figuras 1, 2 e 3), o inverso aconteceu para os valores de COx da fração reativa $\mathrm{C}_{\mathrm{NaOH}}$ (Figuras 5 e 6), ou seja, com o aumento das isóbatas, houve diminuição da capacidade de transferência de elétrons da MO. As Figuras 5 e 6 mostraram que a COx determinada nas amostras ao norte e ao sul variaram de forma semelhante e com taxas próximas em função da profundidade.

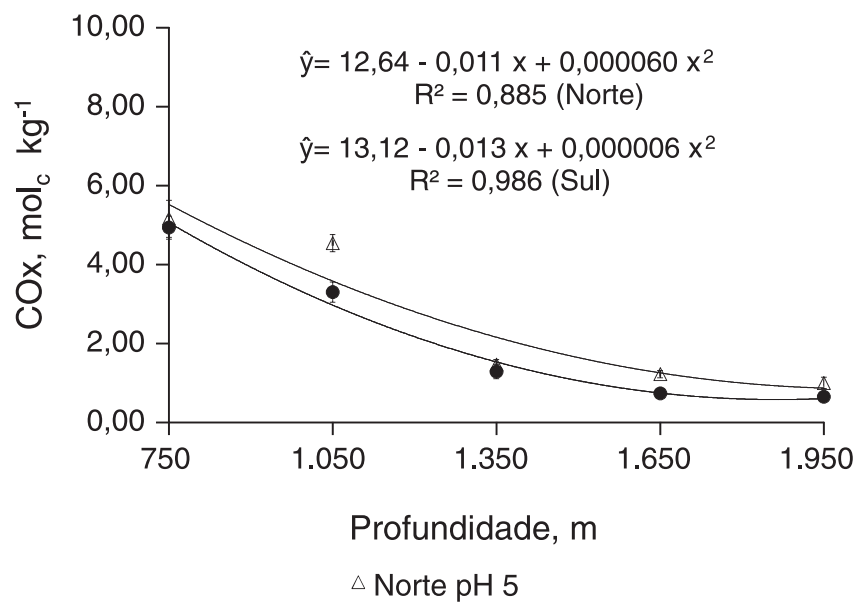

Figura 5. Valores observados para a capacidade de oxidação (COx) a pH 5 da matéria orgânica de oceanos profundos da bacia petrolífera Norte Fluminense corrigidas para as massas de carbono em função da profundidade de amostragem

A relação direta entre a $\mathrm{COx}$ dos ácidos húmicos e a aromaticidade na forma de grupos fenólicos e semiquinonas, determinados, respectivamente, por espectroscopia de ressonância magnética nuclear da $\mathrm{MO}$ do solo $\left({ }^{13} \mathrm{C} \mathrm{RMN}\right)$ e por espectroscopia de ressonância paramagnética de elétrons (RPE) foi verificada por Baldotto et al..$^{28} \mathrm{Assim}$, 
Tabela 5. Matriz de correlações de Pearson para as variáveis estudadas

\begin{tabular}{cccccccc}
\hline & $\delta^{15} \mathrm{~N}$ & $\delta^{13} \mathrm{C}$ & $\mathrm{C}_{\text {Total }}$ & $\mathrm{N}$ & $\mathrm{C} / \mathrm{N}$ & $\mathrm{C}_{\mathrm{NaOH}}$ & $\mathrm{C}_{\mathrm{Residual}}$ \\
\hline $\mathrm{COx} \mathrm{pH} 5$ & $-0,84^{* *}$ & $-0,71^{*}$ & $-0,68^{*}$ & $-0,69^{*}$ & $0,53^{\circ}$ & $\mathrm{NS}$ & $-0,68^{*}$ \\
$\mathrm{COx} \mathrm{pH7}$ & $-0,87^{* *}$ & $-0,73^{* *}$ & $-0,70^{*}$ & $-0,72^{* *}$ & $0,61^{*}$ & $\mathrm{NS}$ & $-0,71^{*}$ \\
\hline
\end{tabular}

NS, $* *, * \mathrm{e}^{\circ}=$ não significativo e significativo a 1,5 e $10 \%$ de probabilidade, respectivamente.

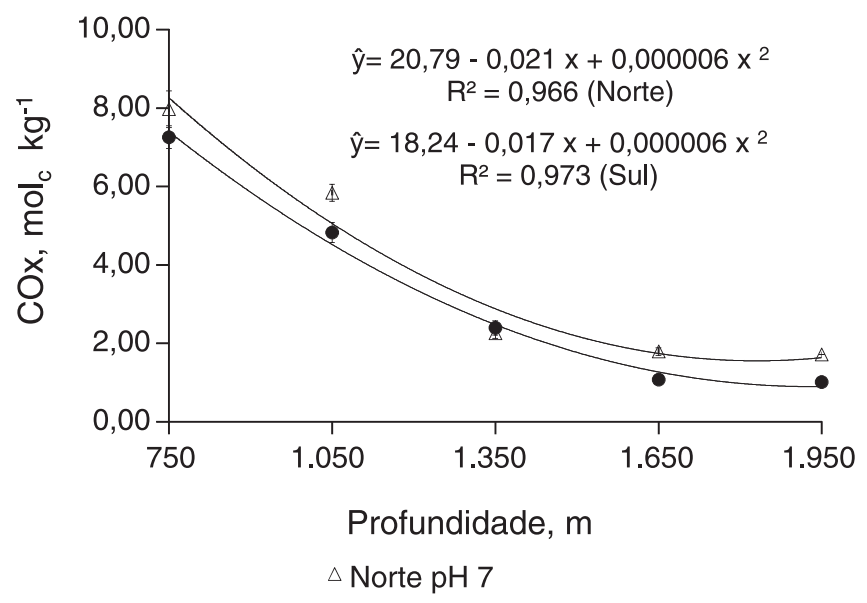

Figura 6. Valores observados para a capacidade de oxidação (COx) a pH 7 da matéria orgânica de oceanos profundos da bacia petrolífera Norte Fluminense corrigidas para as massas de carbono em função da profundidade de amostragem

o aumento concomitante do grau de condensação e da atividade redox dos ácidos húmicos sugere a possibilidade de se estabelecerem modelos preditivos entre a $\mathrm{COx}$ e o grau de estabilidade das $\mathrm{SH}$ e, por conseguinte, estimarem índices de persistência desta forma de $\mathrm{C}$ no sistema. Considerando o exposto sobre estrutura-atividade redox das $\mathrm{SH}$, a respeito dos sedimentos amostrados no presente estudo, pode-se esperar que materiais orgânicos mais aromáticos apresentem menores valores de $\delta^{13} \mathrm{C}, \delta^{15} \mathrm{~N}$ e menores teores $\mathrm{C}_{\text {Total }}$ e $\mathrm{C}_{\text {Residual }}$ e, por outro lado, maior valor da relação $\mathrm{C} / \mathrm{N}$ como observado no Tabela 5 .

Finalmente, A diminuição da COx de acordo com o aumento de $\delta^{13} \mathrm{C}$ e de $\delta^{15} \mathrm{~N}$ e, também, com diminuição da relação $\mathrm{C} / \mathrm{N}$, indica maior aromaticidade na forma de grupos fenólicos e semiquinonas do material orgânico reativo nas amostras de maior isóbata, embora a concentração de carbono orgânico total tenha sido maior nas amostras de águas mais profundas.

\section{CONCLUSÕES}

As substâncias húmicas da matéria orgânica do solo são o maior reservatório de carbono nos sistemas naturais. Em tais sistemas, a qualidade e a estabilidade do carbono podem ser estimadas pelo aumento da concentração das frações humificadas, a qual, entre outros fatores, está condicionada ao balanço entre as perdas e os ganhos envolvendo as reações de oxidação e de redução da matéria orgânica nos ecossistemas. Tanto o fracionamento da matéria orgânica, como a reatividade de suas frações, foram usadas neste estudo para comparar sedimentos de oceanos profundos na Bacia Petrolífera de Campos dos Goytacazes, RJ. As concentrações das formas de C orgânico e nitrogênio estudadas aumentaram com a profundidade amostrada (de $750 \mathrm{~m}$ até $1.950 \mathrm{~m}$ ) na área sul. $\mathrm{Na}$ área norte os valores não diferiram significativamente. As frações definidas operacionalmente como ácidos fúlvicos livres e ácidos húmicos não foram detectadas nas amostras conforme os métodos usados, sendo obtidas as frações ácidos fúlvicos $\left(\mathrm{C}_{\mathrm{NaOH}}\right)$ e huminas $\left(\mathrm{C}_{\mathrm{Residual}}\right)$, sendo essa última a fração a preponderante. Os valores do ${ }^{\mathrm{F}} \mathrm{E}_{\mathrm{H}}{ }^{\mathrm{o}}$ para a semi-reação de redução do par redox $\mathrm{MO}_{\mathrm{Ox}}, \mathrm{MO}_{\mathrm{Red}}$ das amostras coletadas em oceanos profundos da bacia petrolífera de Campos-RJ foram semelhantes, sendo em média de 0,768 V. A COx da MO variou com o pH do sistema e diminuiu com o aumento das isóbatas (de $750 \mathrm{~m}$ até $1.950 \mathrm{~m}$ ), mas variou pouco em relação às amostragens direcionadas ao norte ou ao sul da plataforma. Incrementos na capacidade de oxidação da matéria orgânica, concomitantemente com o aumento das isóbatas, foram acompanhados pela diminuição dos teores de $\delta^{13} \mathrm{C}, \delta^{15} \mathrm{~N}, \mathrm{C}_{\text {Total }}$ $\mathrm{e}_{\text {Residual }}$ e, simultaneamente, pelo acréscimo da relação $\mathrm{C} / \mathrm{N}$.

\section{AGRADECIMENTOS}

O presente trabalho contou com auxílios dos projetos CNPq/ CTHidro Proc. 556160/2006-1/151856/2007-9, CNPq Proc. 420050/2005-1, CNPq/BMBF Proc. 590002/2005-8 e FAPERJ Proc. E-26/153.086/2006 e E- 26/110.633/2007). Os autores agradecem à UENF e à UFV pelo uso da infraestrutura institucional disponível, à Petrobras pela possibilidade de coleta, análise do material e apoio financeiro e à tripulação do navio Astro Garoupa pelo apoio logístico na coleta das amostras.

\section{REFERÊNCIAS}

1. Piccolo, A.; Soil Science 2001, 166, 810.

2. Struyk, Z.; Sposito, G.; Geoderma 2001, 102, 329.

3. Baldotto, M. A.; Canellas, L. P.; Canela, M. C.; Simões, M. L.; Martin-Neto, L.; Fontes, M. P. F.; Velloso, A. C. X.; Revista Brasileira de Ciência do Solo 2007, 3, 465.

4. Baldotto, M. A.; Canela, M. C., Canellas, L. P.; Rezende, C. E.; Velloso, A. C. X.; Revista Brasileira de Ciência do Solo 2008, 2, 1043.

5. Baldotto, M. A.; Rosa, R. C. C.; Maciel, M. S.; Canela, M. C.; Canellas, L. P.; Rezende, C. E.; Velloso, A. C. X.; Quim. Nova 2009, 4, 891

6. Baldotto, M. A.; Canellas, L. P.; Rosa, R. C. C.; Rangel, T. P.; Salomão, M. S. M. B; Rezende, C. E.; Quim. Nova 2011, 6, 973.

7. Martin-Neto, L.; Vieira, E. M.; Sposito, G.; Environ. Sci. Technol. 1994, $28,1867$.

8. Pérez, M. G.; Martin-Neto, L.; Saab, S. C.; Novotny, E. H.; Milori, D. M. B. P.; Bagnato, V. S.; Colnago, L. A.; Melo, W. J.; Knicker, H.; Geoderma 2004, 118, 181.

9. Milori, D. M. B. P.; Martin-Neto, L.; Bayer, C.; Mielniczuck, J.; Bagnato, V. S.; Soil Science 2002, 167, 739.

10. Budziak, C. R.; Maia, C. M. B. F.; Mangrich, A. S.; Quim. Nova 2004, $27,399$.

11. Tossell, J. A. Geochim. Cosmochim. Acta 2009, 73, 2023.

12. Stumm, W.; Morgan, J. J.; Aquatic Chemistry: Chemical Equilibria and Rates in Natural Waters. $3^{\text {th }}$ ed., Wiley: New York, 1996.

13. Sposito, G. The Chemistry of Soils. $2^{\text {th }}$ ed., Oxford University Press: New York, 2008.

14. Helburn, R. S.; Maccarthy, P.; Anal. Chim. Acta 1994, 295, 263.

15. Matthiessen, A.; Vom Wasser 1995, 84, 229.

16. Lovley, D. R.; Coates, J. D.; Blunt-Harris, E. L.; Phillips, E. J. P.; Woodward, J. C.; Nature 1996, 382, 445

17. Scott, D. T.; Mcknight, D. M.; Harris, E.; Kolesar, S.; Lovley, D.; Environ. Sci. Technol. 1998, 32, 2984. 
18. Araujo, B. F.; Almeida, M. G.; Salomão, M. S. M. B; Gobo, R. R.; Siqueira, V. C.; Ovalle, R. R. C.; Rezende, C. E.; Quim. Nova 2010, 3, 501.

19. Thurman, E. M.; Malcolm, R. L.; Environ. Sci. Technol. 1981, 15, 463.

20. Light, T. S.; Anal. Chem. 1972, 44, 1038.

21. Steel, R. G. D.; Torrie, J. H. Principles and Procedures of Statistics. $2^{\text {th }}$ ed., McGraw-Hill: New York, 1980.

22. Kao, S. J.; Shiah, F. K.; Wang, C. H.; Liu, K. K.; Cont. Shelf Res. 2006, $26,2520$.
23. Bartlett, R. J.; James, B. R.; Advances in Agronomy 1993, 50, 151.

24. Bass Becking, L. G. N.; Kaplan, I. R.; Moore, D.; Journal of Geology 1960, 68, 243

25. Alberts, J. J.; Schindler, J. E.; Miller, R. W.; Science 1974, 184, 895.

26. Sunda, W. G.; Kieber, D. J.; Nature 1994, 367, 62.

27. Dobbss, L. B.; Runjaneck, V. M.; Baldotto, M. A.; Velloso, A. C. X.; Canellas, L. P.; Revista Brasileira de Ciência do Solo 2009, 33, 51.

28. Baldotto, M. A.; Canela, M. C.; Canellas, L. P.; Dobbss, L. B.; Velloso, A. C. X.; Revista Brasileira de Ciência do Solo 2010, 34, 1543. 